\title{
Research on the Identification Method and Modeling of Unmanned Aerial Vehicle based on Neural Network
}

\author{
Yang Zhao ${ }^{\mathrm{a}^{*}}$ and $\mathrm{Li} \mathrm{Li}^{\mathrm{b}}$ \\ Department of Mechanical and Electrical Engineering, Guangdong University of Science \& \\ Technology, Dongguan, China \\ azhaoyangmatp@163.com, ${ }^{\mathrm{b}} 65799279 @ q q . c o m$ \\ * The Corresponding Author
}

Keywords: Identification method; Unmanned aerial vehicle; Neural network; Linear model

\begin{abstract}
Because of its unique system features, it is very difficult for small rotor UAVs to be modeled. In this paper, the method of unmanned aerial vehicle modeling is summarized in detail. In order to achieve a good control effect, the accuracy of the model is very important for the design and verification of the control law. In this paper, the linear model and nonlinear model identification method and identification algorithm of unmanned aerial vehicle are proposed. The simulation results show that the attitude control precision is improved effectively.
\end{abstract}

\section{Introduction}

Compared to its full-size counterpart, Unmanned Aerial Vehicle (UAV) is much cheaper, easier to carry and more agile, and especially the rotary-wing class, with its ability of hovering, taking off and landing vertically, is very suitable for flying in low-altitude and cramped space, so it is expected to be used widely both in military and civilian domain in the future.

\section{The Identification Model of UAV}

The Linear Model of UAV. The dynamics of UAV are divided into horizontal dynamic (attitude dynamics), vertical dynamic and yaw dynamics. The main rotor's swing has a relatively large dynamic impact on the horizontal. When the vehicle is hovering, its dynamic behavior can be expressed as two first order models coupled vertically and horizontally.

$$
\left\{\begin{array}{l}
\dot{a}_{1 s}=-\frac{a_{1 s}}{\tau_{f}}-q+A_{b 1 s} b_{1 s}+A_{\text {lat }} \delta_{\text {lat }}+A_{\text {lon }} \delta_{\text {lon }} \\
\dot{b}_{1 s}=-\frac{b_{1 s}}{\tau_{f}}-p+B_{a 1 s} a_{1 s}+B_{l a t} \delta_{l a t}+B_{l o n} \delta_{l o n}
\end{array}\right.
$$

Considering the coupling of the rotor and the fuselage, the simplified model of the horizontal dynamic linearization is .

$$
\left\{\begin{array}{l}
\dot{p}=L_{u} u+L_{v} v+L_{b 1 s} b_{1 s}+L_{a 1 s} a_{1 s} \\
\dot{q}=M_{u} u+M_{v} v+M_{b 1 s} b_{1 s}+M_{a 1 s} a_{1 s} \\
\dot{u}=X_{v} v-g \theta+X_{a 1 s} a_{1 s} \\
\dot{v}=Y_{v} v+g \phi+Y_{b 1 s} b_{1 s}
\end{array}\right.
$$

Vertical dynamic representation

$$
\dot{z}=Z_{w} w+Z_{c o l} \delta_{c o l}
$$

Yaw dynamics can be expressed as 


$$
\begin{aligned}
& \dot{r}=N_{r} r+N_{p e d}\left(\delta_{p e d}-r_{f b}\right) \\
& \dot{r}_{f b}=-K_{r f b} r_{f b}+K_{r} r
\end{aligned}
$$

Among them, $a_{1 s}, b_{1 s}$ is the waving corner of the main rotor. $p, q, \phi, \theta$ represents roll angle rate, pitch angle rate, roll angle and pitching angle. $u, v, z, w$ represents the transverse velocity, longitudinal velocity, vertical displacement and vertical velocity. $r, r_{f b}$ is yaw angular rate and yaw rate feedback. $\delta_{\text {lat }}, \delta_{l o n}, \delta_{\text {ped }}, \delta_{\text {col }}$ is lateral cyclic pitch, pitch, tail rotor control inputs and the total distance of longitudinal cycle.

The state space model is set up as follows

$$
\dot{x}=A x+B u
$$

Among them, $x=\left[u, v, p, q, \phi, \theta, a_{1 s}, b_{1 s}, w, r, r_{f b}\right]^{T}$ is state variable, $u=\left[\delta_{l a t}, \delta_{l o n}, \delta_{p e d}, \delta_{c o l}\right]^{T}$ is control input. $A$ and $B$ is

$$
A=\left[\begin{array}{ccccccccccc}
X_{u} & 0 & 0 & 0 & 0 & -g & X_{a 1 s} & 0 & 0 & 0 & 0 \\
0 & Y_{v} & 0 & 0 & g & 0 & 0 & Y_{b 1 s} & 0 & 0 & 0 \\
L_{u} & L_{v} & 0 & 0 & 0 & 0 & L_{a 1 s} & L_{b 1 s} & 0 & 0 & 0 \\
M_{u} & M_{v} & 0 & 0 & 0 & 0 & M_{a 1 s} & M_{b 1 s} & 0 & 0 & 0 \\
0 & 0 & 1 & 0 & 0 & 0 & 0 & 0 & 0 & 0 & 0 \\
0 & 0 & 0 & 1 & 0 & 0 & 0 & 0 & 0 & 0 & 0 \\
0 & 0 & 0 & -1 & 0 & 0 & -1 / \tau_{f} & 0 & 0 & 0 & 0 \\
0 & 0 & -1 & 0 & 0 & 0 & B_{a 1 s} & -1 / \tau_{f} & 0 & 0 & 0 \\
0 & 0 & 0 & 0 & 0 & 0 & Z_{a 1 s} & Z_{b 1 s} & Z_{w} & Z_{r} & 0 \\
0 & 0 & N_{p} & 0 & 0 & 0 & 0 & 0 & N_{w} & N_{r} & M N_{p e d} \\
0 & 0 & 0 & 0 & 0 & 0 & 0 & 0 & 0 & K_{r} & M K_{r f b}
\end{array}\right] \quad B=\left[\begin{array}{cccc}
0 & 0 & 0 & 0 \\
0 & 0 & 0 & 0 \\
0 & 0 & 0 & 0 \\
0 & 0 & 0 & 0 \\
0 & 0 & 0 & 0 \\
0 & 0 & 0 & 0 \\
A_{l a t} & A_{\text {lon }} & 0 & 0 \\
B_{l a t} & B_{\text {lon }} & 0 & 0 \\
0 & 0 & 0 & Z_{c o l} \\
0 & 0 & N_{p e d} & N_{c o l} \\
0 & 0 & 0 & 0
\end{array}\right]
$$

Due to the dominant control role of main rotor, small UAV is more suitable for system identification. Therefore, the identification of full-size and small UAV is necessary. The linear time invariant model and maneuver mode is shown in Fig. 1.

quick turn 2

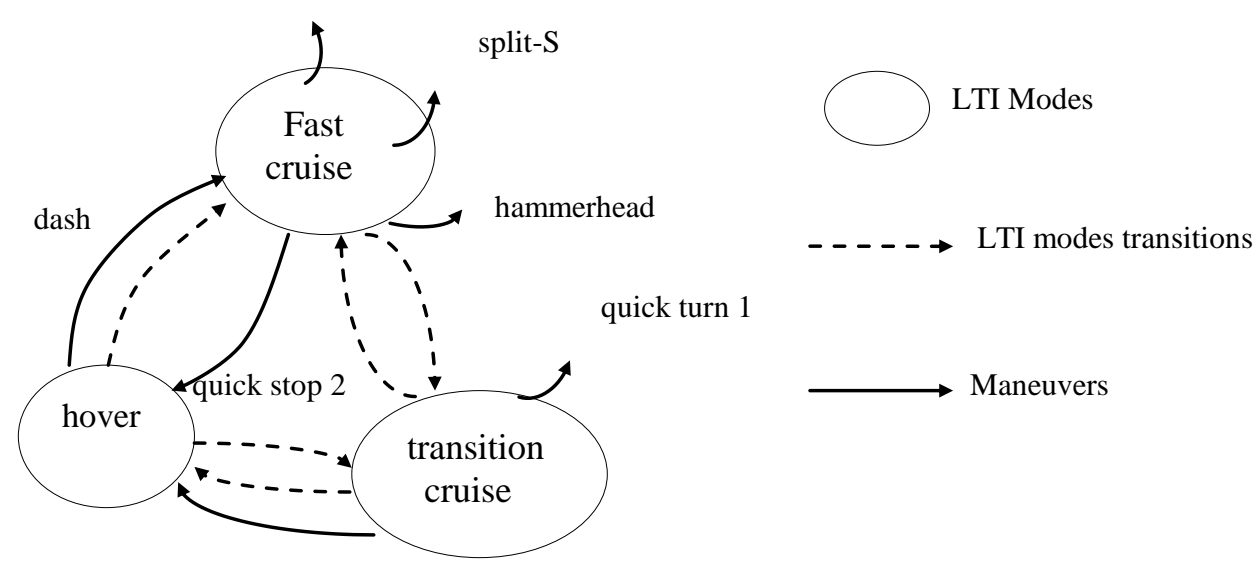

Figure 1. Finite The linear time invariant model and maneuver mode and its conversion

The non-linear model. A discrete NLARX model with $r$ input and $m$ output is as follows $\mathrm{y}_{\mathrm{m}}(\mathrm{t})=N\left[\mathrm{y}_{\mathrm{m}}(\mathrm{t}-1), \mathrm{y}_{\mathrm{m}}(\mathrm{t}-2), \ldots, \mathrm{y}_{\mathrm{m}}\left(\mathrm{t}-\mathrm{n}_{\mathrm{a}}\right)\right.$, 


$$
\left.\mathrm{u}_{\mathrm{r}}(\mathrm{t}), \mathrm{u}_{\mathrm{r}}\left(\mathrm{t}-\mathrm{n}_{\mathrm{k}}\right), \ldots, \mathrm{u}_{\mathrm{r}}\left(\mathrm{t}-\mathrm{n}_{\mathrm{k}}-\mathrm{n}_{\mathrm{b}}+1\right)+\mathrm{e}_{\mathrm{m}}(\mathrm{t})\right]
$$

Among them, $\mathrm{y}_{\mathrm{m}}(\mathrm{t})=\left[\begin{array}{c}y_{1}(t) \\ \vdots \\ y_{m}(t)\end{array}\right], \mathrm{u}_{\mathrm{r}}(\mathrm{t})=\left[\begin{array}{c}u_{1}(t) \\ \vdots \\ u_{r}(t)\end{array}\right], \mathrm{e}_{\mathrm{m}}(\mathrm{t})=\left[\begin{array}{c}e_{1}(t) \\ \vdots \\ e_{m}(t)\end{array}\right]$ is the system output, input and modeling error. $\mathrm{n}_{\mathrm{a}}$ and $\mathrm{n}_{\mathrm{b}}$ represent the output and input time matrices of the time. $\mathrm{n}_{\mathrm{k}}$ is a time delay matrix from each input to the output. $N[]$ represents the nonlinear mapping of positions.

\section{The Identification Algorithm of UAV}

Set the solving path domain as $30 \mathrm{~km} \times 30 \mathrm{~km}$, randomly set the initial point and the target point of the solving, set the condition as obstacle avoidance path, and set the reference height as 0 . The algorithm flow is shown in Fig. 2 as follows.

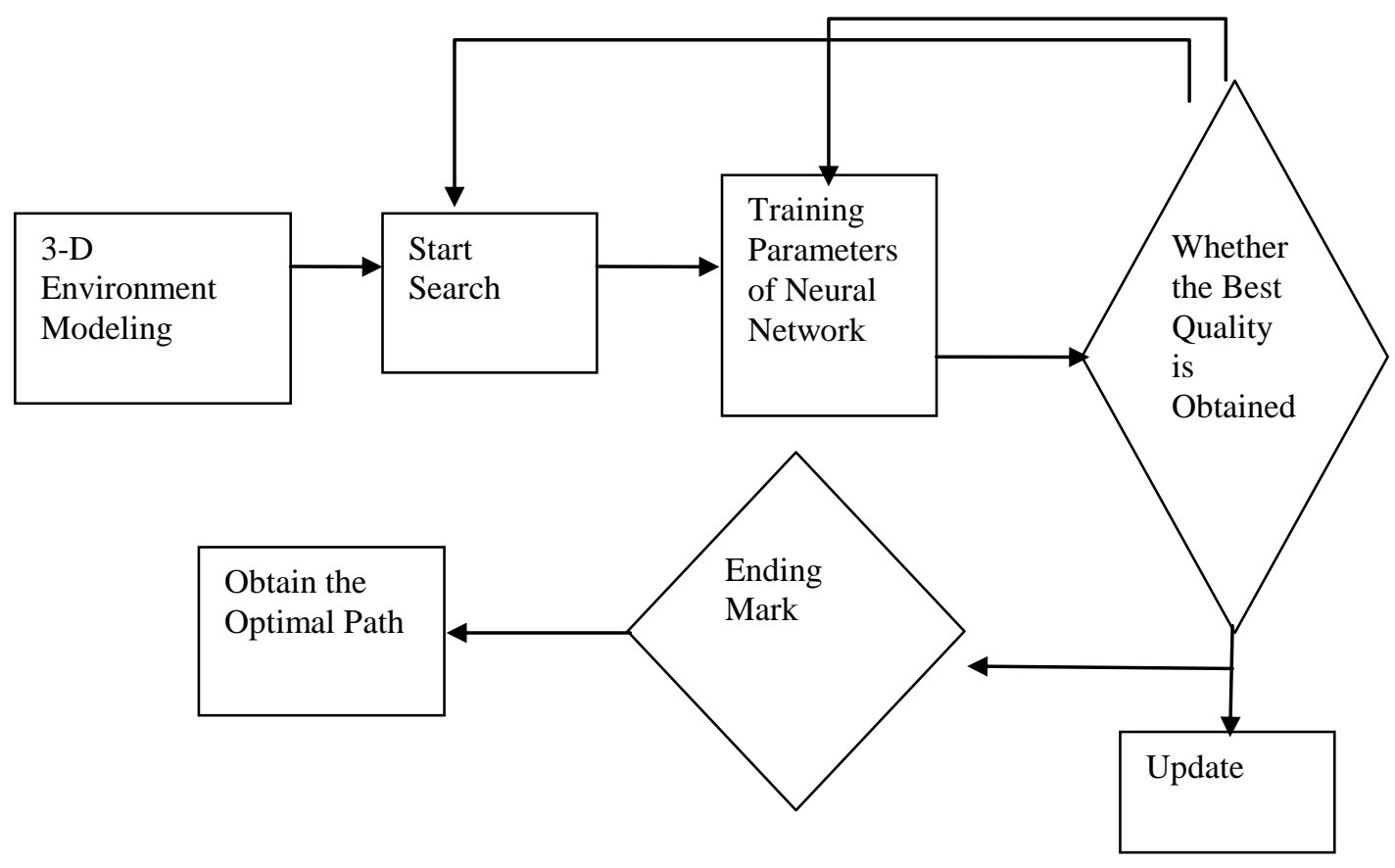

Figure 2. Finite The algorithm flow

The purpose of identification is to get the mapping. The common tool is the neural network, and its structure is like Fig. 3.

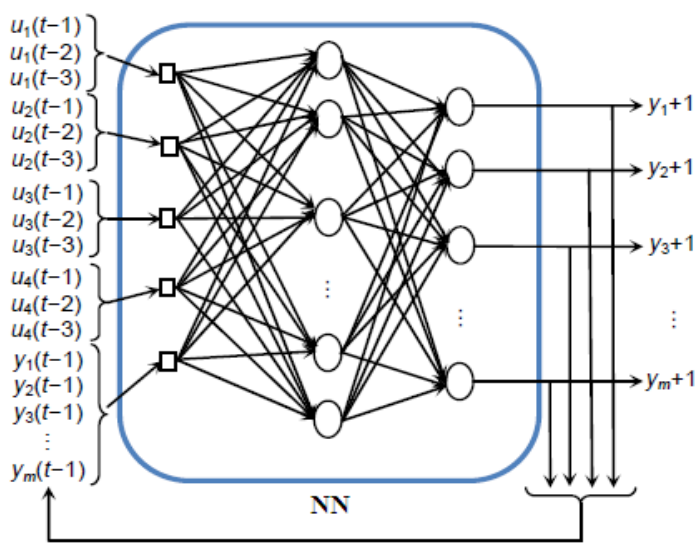

Figure 3. Finite Identification of NLARX model based on neural network 


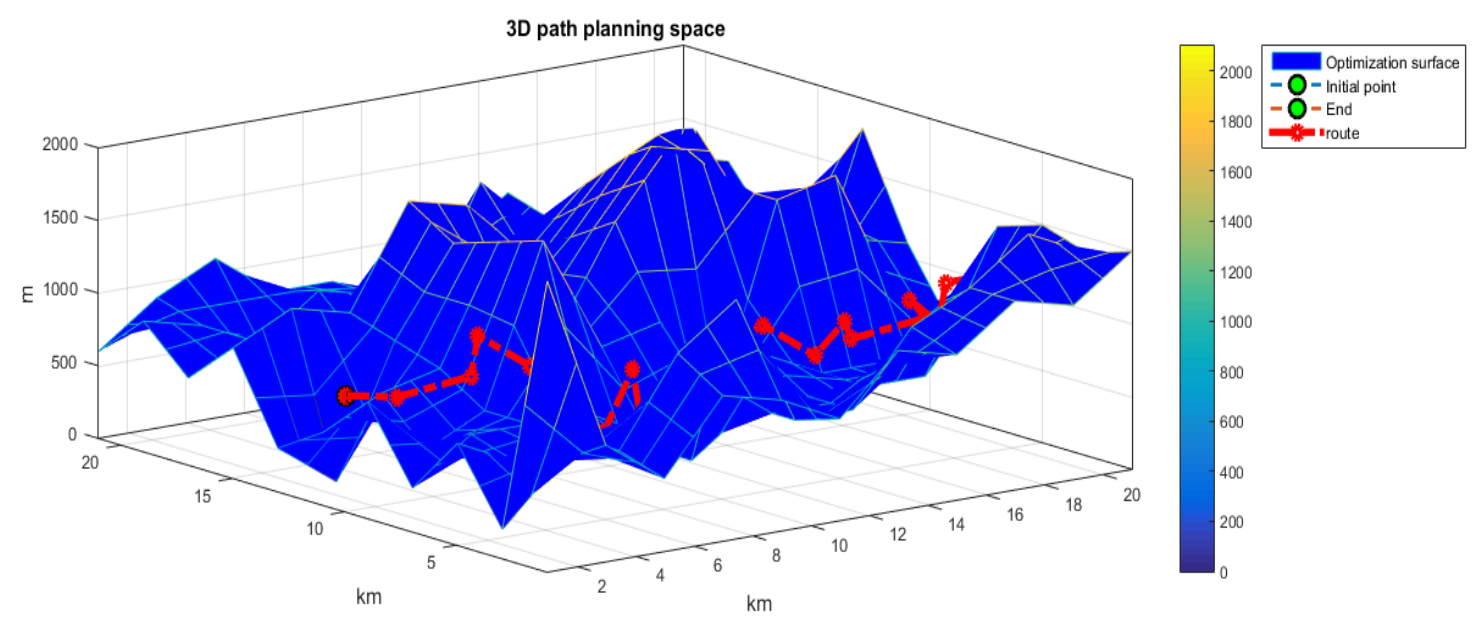

Figure 4. Finite Three-dimensional path graph

Fitness change of algorithm is shown in Fig. 5.

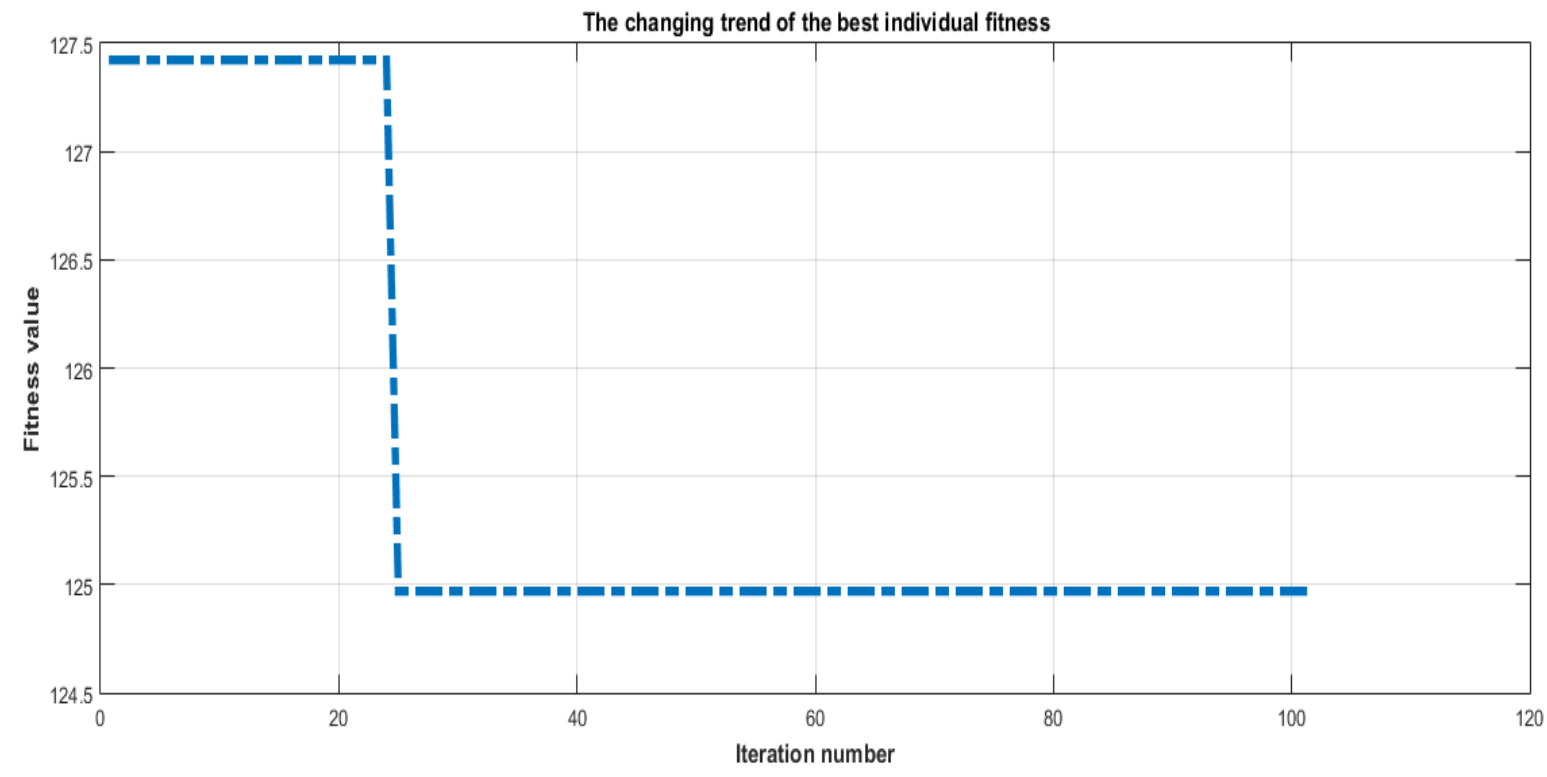

Figure 5. Finite The fitness curve of algorithm

\section{Summary}

This paper is mostly concerned with controller design for UAV based on its six degree of freedom dynamics. First, it gives a brief introduction to the main parts comprising UAV platform and the function of each part, and then analyzes the characteristics of the system and difficulty in designing a controller. After that, a comprehensive review and comparison is made on various modeling and control techniques.

\section{References}

[1] Laura Pozueco, Xabiel García Pañeda, and Roberto García. Adaptable System Based on Scalable Video Coding for High-quality Video Service, Computers \& Electrical Engineering, 39 (2013), 775-789.

[2] Jason Kimball, Tom Wypych, and Falko Kuester. Low Bandwidth Desktop and Video Streaming for Collaborative Tiled Display Environments, Future Generation Computer Systems, 54(2016), 336-343. 
[3] Santiago González, Wilder Castellanos, and Paola Guzmán. Simulation and Experimental Testbed for Adaptive Video Streaming in Ad Hoc Networks, Ad Hoc Networks, 52 (2016), 89-105.

[4] L Wang, Z Yang, and LJ Xu. NCVCS: Network-coding-based Video Conference System for Mobile Devices in Multicast Networks, Ad Hoc Networks, 45 (2016), 13-21.

[5] Georgios Karopoulos, Paolo Mori, and Fabio Martinelli. Usage Control in SIP-based Multimedia Delivery, Computers \& Security, 39 (2013), pp. 406-418.

[6] Alfonso Sandoval Rosas, and José Luis Alejos Martínez. Videoconference System Based on WebRTC With Access to The PSTN, Electronic Notes in Theoretical Computer Science, 329(2016), 105-121.

[7] XH Li, CH Ren, and ML Yue. A Distributed Real-time Database Index Algorithm Based on B+ Tree and Consistent Hashing, Procedia Engineering, 24(2013), 171-176.

[8] ZL Ning, Y Huang, and L Guo. A Channel Estimation Based Opportunistic Scheduling Scheme in Wireless Bidirectional Networks, Journal of Network and Computer Applications, 39 (2014), 61-69.

[9] Ali Moussaoui, and Abdallah Boukeream. A Survey of Routing Protocols Based on Link-stability in Mobile Ad Hoc Networks, Journal of Network and Computer Applications, 47(2015), 1-10.

[10] SC Ye, MY Su, and HH Chen. An Efficient and Secure Approach for A Cloud Collaborative Editing, Journal of Network and Computer Applications, 30(2013), 1632-1641. 